\section{凹局Universidad Francisco 四 de Paula Santander Ocaña - Colombia \\ Vigilada Mineducación}

ISSN: 2422-1783

Nombre de la Revista, Vol. 6. Mes Enero - Junio

\title{
IMPORTANCIA DEL CONTROL DE CALIDAD SOBRE LAS EMPRESAS AUDITORAS NIA 220
}

\section{IMPORTANCE OF QUALITY CONTROL ABOUT NIA 220 AUDIT COMPANIES}

\author{
Ramiro Gamboa Suarez ${ }^{1}$, Luis A Jiménez R. ${ }^{2}$ \\ 1 Grupo TECNNOSALUD, Docente - Investigador de la Universidad Surcolombiana, Neiva - Huila-Colombia. ORCID: \\ http://orcid.org/0000-0002-5727-6409,E-mail: rgamboa1@uniminuto.edu.com \\ 2 Grupo GTN, Docente - Investigador de la Universidad Colegio Mayor de Cundinamarca - Colombia. ORCID: http://orcid.org/0000- \\ 0001-8656-9406, E-mail: lualf0115@gmail.com
}

Fecha de recibido: 29 de agosto de 2016

Fecha aprobación: 3 de octubre de 2016

Resumen: El presente artículo pretende identificar y analizar el conocimiento de diferentes conceptos de calidad, control, la importancia de la calidad de las firmas auditoras a la hora de prestar sus servicios profesionales; tomando como referencia las normas de calidad según estándares internacionales en la auditoría de estados financieros. Lo anterior con el fin de evaluar la ejecución del trabajo tanto del auditor como de las firmas auditoras, donde se busca ver competencias y responsabilidades para establecer sistemas de control que garanticen información financiera de calidad, para entender estos procesos se establece como punto de partida la Norma Internacional De Auditoria 220 y algunas interpretaciones internacionales que permiten tener la visión holística sobre los procesos y metodologías inherentes a la ejecución, desarrollo y sistema de control de calidad de las firmas auditoras.

Palabras claves: Auditoria, imagen fiel, dictamen, Escepticismo profesional, calidad, control.

\begin{abstract}
This article aims to identify and analyze the knowledge of different concepts of quality, control, the importance of the quality of audit firms when providing their professional services; taking as reference the quality standards according to international standards in the audit of financial statements. The foregoing in order to evaluate the execution of the work of both the auditor and the auditing firms, where it is sought to see competencies and responsibilities to establish control systems that guarantee quality financial information, to understand these processes the starting point is established International Audit Standard 220 and some international interpretations that allow us to have a holistic view of the processes and methodologies inherent in the execution, development and quality control system of the audit firms.
\end{abstract}

Keywords: Audit, faithfulimage, opinion, professionals kepticism, quality, control. 


\section{INTRODUCIÓN}

La globalización ha generado la integración económica entre los distintos países del mundo, buscando la generación de información confiable y transparente en un lenguaje colectivo, ante los diferentes escenarios y usuarios de la información, en términos de esta situación se puede expresar que la auditoría genera un grado de confianza, seguridad razonable y cumplimiento de propósitos en común. Con la llegada de la ley 1314 del 13 de julio de 2009, se regulan los principios y normas de contabilidad e información financiera y de aseguramiento de la información, de aceptación en Colombia. A partir de ese momento, el país entra en un proceso de convergencia hacia los estándares internacionales de la información financiera, y con ello proporcionalmente, la necesidad de establecer un marco normativo referente a trabajos de auditoría, de revisión y aseguramiento de la información financiera, con observancia de las normas internacionales.

Es así como a partir del 20 de febrero de 2015, con el Decreto 302 expedido por el Ministerio de Comercio, Industria y Turismo, entra en rigor el marco técnico normativo para las normas de aseguramiento de la información, para efectos del presente trabajo, se tratan dos normas contenidas en este decreto, una que referencia al control de calidad para una auditoría de estados financieros NIA 220 y otra que establece el control de calidad para firmas que realizan auditorías y revisiones de estados financieros y otros compromisos de aseguramiento y servicios relacionados - NICC1.

Al Hablar del control de calidad sobre las firmas auditoras, se requiere inicialmente verificar los sistemas de control existentes en la práctica profesional del Auditor, puesto que dichos sistemas permiten identificar los posibles riesgos inherentes al desarrollo del encargo de trabajo del auditor o la firma auditora. (CAIGG, 2015).

\section{MARCO CONCEPTUAL}

\section{Actividad de Auditoría:}

Una actividad de aseguramiento, consultoría, seguimiento, rutinarias y/o obligatorias por ley o solicitudes realizadas por el Jefe de Servicio, que está contenida en el Plan Anual de Auditoría de una organización gubernamental, aprobado por la Jefatura del Servicio. (CAIGG, 2015).

\section{Alcance:}

Corresponde a la definición de las materias, muestras y montos a revisar, los límites de la auditoría, el período que se considerará para evaluación en el trabajo y los lugares físicos o virtuales incluidos en el trabajo. (CAIGG, 2015).

\section{Evidencia de Auditoría:}

Es la información que obtiene el auditor interno para respaldar los hallazgos, recomendaciones, opiniones y conclusiones del trabajo. Equivale a la convicción que le asiste al auditor de que "una cosa" es tal cual como él la ha percibido, luego de practicar el examen analítico de dicha cosa, mediante la aplicación de los procedimientos de auditoría más adecuados en relación con el objetivo perseguido. (CAIGG, 2015).

\section{Fraude:}

Cualquier acto ilegal caracterizado por engaño, ocultación o violación de confianza. Estos actos no requieren la aplicación de amenaza de violencia o de fuerza física. Los fraudes son perpetrados por individuos y por organizaciones para obtener dinero, bienes o servicios, para evitar pagos o pérdidas de servicios, o para asegurarse ventajas personales o de negocio. (CAIGG, 2015).

Programa de Auditoría o Programa de Trabajo:

Documento que tiene como finalidad orientar el trabajo para cumplir con los objetivos de 
auditoría. En este documento se establecen entre otros; los riesgos, controles y pruebas de auditoría a aplicar en terreno. (CAIGG, 2015).

\section{La independencia:}

Constituye un principio que se establece en las diferentes normas que se relacionan con el ejercicio de la auditoría, constituye un factor importante para el desarrollo del trabajo de auditoría en todas sus etapas. Respecto a la independencia (García, Vico, \&Zorio, 2003) diferencian la independencia como real y aparente considerando que el auditor no solo debe considerarse como independiente, sino que sus actuaciones deben demostrar que lo es; por lo tanto, su sentido de independencia debe estar enmarcada dentro de su ser para que pueda ser expresada al exterior. (Corredor, 2015)

\section{CALIDAD}

Antes de dar a conocer la calidad sobre las firmas auditoras, se debe saber que cuando la entidad usuaria requiere de los servicios de profesionales externos, siempre busca obtener buenos resultados sobre el encargo y seguridad sobre los controles de esta, que la firma auditora implemente conductas de ética profesional y de la misma manera la firma auditora deberá satisfacer las necesidades que busca la entidad usuaria.

Al Hablar de calidad, se requiere inicialmente verificar los sistemas de control existentes en la práctica profesional del Auditor, puesto que dichos sistemas permiten identificar los posibles riesgos inherentes al desarrollo del encargo (trabajo del auditor). Por lo tanto, existen diversas doctrinas acerca de la importancia de la calidad en la auditoria en lo relacionado a su mejora y mantenimiento, dando así directrices iniciales para la implementación voluntaria de dichas prácticas profesionales en función del papel de auditor. (Escobar, 2015)

Aristóteles consideraba a la calidad como la tercera categoría de las especies. Una costumbre entre los romanos consistía en que el ingeniero que diseñaba un arco se colocaba debajo de él en el preciso momento en que se ponía la última piedra, como compromiso de calidad por lo diseñado y consecuente responsabilidad. Urbano, (2011)

Según Konja, (2003) El principio fundamental que debe cumplir el programa de calidad consiste en la necesidad de que esté diseñado para medir el desempeño de los profesionales en los trabajos asignados de manera objetiva, para identificar oportunidades de mejora en la calidad de dichos trabajos, (Pizarro \& Pozo, 2017).

La Sociedad Americana para el Control de Calidad, (ASQC), considera la calidad como la totalidad de las funciones y características de un producto o servicio, dirigidas a satisfacer las necesidades de cierto usuario.

Para la Organización Europea para el Control de Calidad, (EOQC), la calidad de un producto o servicio representa el grado en que se cubren las exigencias del cliente al cual va destinado y es el resultado de la calidad del diseño y la calidad de la producción, Velásquez (2004).

Previa consulta con todos los socios y empleados, una firma de auditoría puede optar por identificar los valores comunes que está dispuesta a respaldar y que serán parte de su manual de control de calidad. Estos valores pueden incluir calidad del servicio, comunicaciones oportunas y adecuadas con los clientes y una actitud profesional colegiada dentro de la firma de auditoría, que debe estar respaldada por su integridad, diligencia y a consultas.

Es importante que los empleados interpreten que la cultura interna premia el desempeño y el trabajo de calidad. Los empleados deben tener claro estos mensajes a través de todo tipo de comunicaciones, como la declaración de misión y objetivos de la firma de auditoría, la formación interna y externa y el diálogo con el (los) socio (s) de la firma de auditoría. (IFAC,2011). 
Por otra parte, reuniendo cada uno de los conceptos de calidad se puede decir que las firmas auditoras deben fomentar una cultura interna de calidad en la presentación de cada una de sus funciones y también se debe tener en cuenta que el personal quien lidera las funciones este constantemente ilustrándose para que de la misma manera el desempeño sea el esperado.

Es muy importante que el auditor o socio del encargo, implemente sobre la auditoria un plan debidamente específico para el desarrollo de los objetivos teniendo en cuenta que se pueden presentar diversas situaciones por lo cual no siempre se tiene un mismo plan de trabajo.

\section{CONTROL}

El control va de la mano con el poder y atención que se puede llegar a tener sobre sobre cierto proyecto o actividad a realizar, La manera en que podemos tener cierto dominio sobre el desarrollo del mismo y ciertamente se busca el cumplimiento de metas sean a corto, mediano o largo plazo es ahí donde la empresa usuaria, debe conocer la entidad que prestara los servicios de auditoría.

Se pueden decir que hay varios conceptos de control entre ellos los siguientes

La norma ISQC 1 se enfoca en el control de la calidad a nivel de firma, es por ello que el objetivo de la firma es establecer y mantener un sistema de control de la calidad para darle una seguridad razonable de que: (1) la firma y su personal cumplen con las normas profesionales y con los requerimientos legales y regulatorios que apliquen; y (2) los informes emitidos por la firma o los socios por contrato se encuentran en armonía con las circunstancias, (Estrada, 2015).

Según Taylor \& Glezen (1997), el control es un proceso sistemático que consiste en obtener y evaluar objetivamente evidencia sobre las afirmaciones relativas a los actos y eventos de carácter económico; con el fin de determinar el grado de correspondencia entre las afirmaciones y los criterios establecidos, para luego comunicar los resultados a las personas interesadas, (García \& Montaño, 2016)

De esta manera el control de calidad sobre las firmas auditoras que debe hacer la empresa usuaria es primeramente un estudio del caso antes de ser contratada la firma, pues la empresa usuaria debe asegurarse que la firma auditora que contratara para ejercer la auditoria tenga la debidamente certificación de calidad entre ellas la ISO 9001, que se encarga de garantizar la calidad de los productos y servicios de las empresas, de este modo será fiable la opinión que pueda expresar sobre la auditoria.

\subsection{CONTROL DE CALIDAD DE UNA EMPRESA}

Cuando una empresa logra tener el control de calidad de sus bienes o servicios, está logrando satisfacer las expectativas del cliente lo cual nos puede arrojar buenas estadísticas del funcionamiento y nos muestra que el plan de acción está dando frutos.

En este caso el plan de acción es aquel que se plantea a la hora de hacer la auditoria diferenciando las expectativas de cada entidad usuaria.

"Los auditores tienen un papel fundamental para contribuir a la credibilidad de los estados financieros sobre los que reportan. Las auditorías de calidad ayudan la estabilidad financiera. Como responsables del establecimiento de las normas de auditoría a nivel global, tenemos la responsabilidad de velar por el interés público y establecer la normativa y la guía para que los auditores realicen auditorias de alta calidad, que a su vez refuercen la fiabilidad y confianza en los estados financieros y en la información financiera en el sentido más amplio", (Borrás,1914). 
La intención del Código de Ética Internacional IFAC es que sirva como modelo sobre el cual se basen las orientaciones éticas nacionales y establece estándares de conducta para los contadores profesionales y señala los objetivos y principios fundamentales que deben ser observados por parte de los contadores profesionales en orden a lograr los objetivos comunes, es lamentable la falta de credibilidad de las contabilidades a nivel empresarial y la cantidad de fraudes descubiertos recientemente en múltiples empresas lo que ha puesto en juicio la calidad de ética de la contaduría pública, (Mantilla, S. 2002).

En cumplimiento de dicha misión, el Consejo de la IFAC ha establecido el Consejo de Normas Internacionales de Ética para Contadores cuya finalidad es desarrollar y emitir, bajo su propia autoridad, normas de ética de alta calidad y otros pronunciamientos, para que sean utilizados por los profesionales de la contabilidad en todo el mundo, (IFAC, 2009).

El código IFAC establece los principios fundamentales de la ética profesional, que incluye:

- Integridad

- Objetividad

- Competencia profesional y cuidado debido

- Confidencialidad

- Conducta profesional

Por lo anterior, dentro de los principios fundamentales del Código de Ética, se debe "mantener el conocimiento y la aptitud profesional al nivel necesario para asegurar que el cliente o la entidad para la que trabaja reciben servicios profesionales competentes basados en los últimos avances de la práctica, de la legislación y de las técnicas y actuar con diligencia y de conformidad con las normas técnicas y profesionales aplicables. (IFAC, 2010).
Cabe recordar que en las normas internacional se destaca como concepto clave "firma" la cual se define como "un profesional ejerciente individual, una sociedad cualquiera que sea su forma jurídica, o cualquier otra entidad de profesionales de la contabilidad".

Mientras que en Colombia se actúa como firma un contador público como persona natural o bien una sociedad de contadores públicos, por lo que el alcance la NICC1 hace referencia a la responsabilidad que la firma tiene sobre el control de calidad de sus trabajos, bien sobre auditorías, revisiones de estados financieros $\mathrm{u}$ otros compromisos de aseguramiento, por lo tanto los procedimientos de control dependerán de su tamaño y características, es decir dar certeza de que todos sus profesionales cumplen con los requerimientos legales y regulatorios fijados en marcos internacionales.

Podemos decir, que la calidad de una auditoria se logra con los esfuerzo y compromiso que hace el equipo de trabajo, bien con la observancia de actitudes éticas, una competencia profesional y documentos o papeles debidamente preparados, así como la aplicación rigurosa de sus procesos de control, así como el tiempo asignado para cada trabajo de auditoría.

Hay las IFAC con la misión que tiene, quiere que todos los contadores públicos sean siempre éticos profesionalmente en el momento de realizar el trabajo de Auditoria o de cualquier otra área contable.

\section{NORMAS INTERNACIONALES DE AUDITORIA - NIA}

La aparición de las Normas Internacionales de Auditoria (NIA) son elaboradas por la Federación Internacional de Contadores (IFAC), las cuales son actualizadas año a año por el Comité Internacional de Prácticas de Auditoria, donde buscan un lenguaje internacional del buen manejo del mundo contable, estas normas estandarizan 
los procesos internos de las organizaciones, permitiendo que estas evolucionen en la calidad de sus contabilidades y los procedimientos que estas aplican a la misma área, (Castro, 2015).

Las Normas Internacionales de Auditoría deben ser aplicadas, en forma obligatoria y deben aplicarse también, con la adaptación necesaria, y es obligación de los contadores brindar esta labor con calidad y trasparencia, por ello este ensayo brindaría los conocimientos de las normas de manera oportuna y confiable las cuales sean aplicables en el momento de hacer la auditoria a una empresa.

Las Normas Internacionales de Auditoria contiene unos conceptos, requerimientos y una guía de aplicación y otras anotaciones explicativas que tienen como finalidad ayudar al auditor a alcanzar una seguridad razonable ya que eso le permite estar libre de incorreciones materiales, también le permitirá obtener un mayor grado de confianza en la información que se le entrega a los usuarios.

En la parte de las firmas de auditoría podemos ver que la obligatoriedad de las implementaciones de normas internacionales, se da una voz de alerta para que las firmas de auditoría pongan su mirada y muestre un alto grado de interés para la necesidad de adoptar las Normas Internacionales de Auditoria en sus procedimientos.

Es importante también destacar que las normas tienen dos subdivisiones una de ellas es la NIA que corresponde a los servicios de auditoría, y por otro lado están las NIA/SAS, que son los servicios afines de la norma, esta representa las acciones de revisión de procesos pertenecientes a la compilación de la información, es de apuntar que la revisión hace parte fundamental de la auditoria, en este punto el auditor puede expresar sus conceptos que le generen incertidumbre, referentes a los inconvenientes o errores de la información extraída hasta el momento.

\subsection{CONTROL DE CALIDAD DE LA AUDITORIA EN LOS ESTADOS FINANCIEROS - NIA 220}

El punto de partida para el estudio de la NIA 220, está en la identificación de las responsabilidades del auditor con respecto a los procedimientos de Calidad en la auditoria de estados Financieros y la responsabilidad del equipo de encargo, cuando sea el caso. Las normas difieren en que la NIA especifica que es responsabilidad del socio auditor y su equipo de trabajo implementar procedimientos de control de calidad que sean aplicados al compromiso de auditar estados financieros, mientras que la NICC1 identifica a la firma como responsable de establecer un sistema de control de calidad que transmita confianza a los equipos de trabajo del compromiso, de manera que los procedimientos aplicados al compromiso con referencia al control de calidad dando seguridad del cumplimiento de normas legales y profesionales, al igual que seguridad en el informe del auditor, conforme a las actividades y hechos auditados.

Esta NIA 220, presenta como objetivo del auditor la implementación de procesos de Control de Calidad, que puedan proporcionar al auditor una seguridad razonable, respecto al cumplimiento de estándares profesionales, requerimientos legales y regulatorios aplicables al ente auditado y con la razonabilidad que el informe emitido por el auditor es apropiado a las circunstancias y al entorno de la entidad.

Esta NIA - 220 la calidad de trabajo del auditor se basa de unas buenas prácticas de auditoria, con el fin de dar seguridad en los procedimientos que se apliquen a cualquier trabajo que realice en particular proporcionando una seguridad razonable en el momento del cumplimiento de las normas profesionales

\section{ASPECTOS GENERALES DE LA NIA 220}

\section{Requisitos}


- Responsabilidades de liderazgo en la calidad de las auditorias: Es el socio encargado de la calidad global del proceso de auditoría, el encargo de auditoria, por lo tanto, se debe desarrollar conforme a las normas profesionales y disposiciones legales existentes para las pymes en Colombia. Partiendo con la premisa que la Calidad es el eje fundamental de la auditoria, no debe existir entonces reserva de la información y los informes deben estar libres de limitaciones, por lo que deben estar acordes a las circunstancias auditadas.

- Requerimientos de ética: debe existir una inspección oportuna al equipo de auditoría, para el cumplimiento del código de ética, previsto como norma legal y profesional de obligatorio cumplimiento. Sumado a esto, se debe mantener como pilar central de la auditoria de calidad el principio de independencia y tomar las decisiones o medidas adecuadas, en cuanto a políticas y procedimientos para el cumplimiento de dicho principio, tomando como referencia las salvaguardas mencionadas en el Código de ética de la IFAC, con el fin de reducir los riesgos y amenazas inherentes a su cumplimiento.

- Aceptación y continuación de las relaciones con el cliente y de los encargos específicos: como se estipula en la NICC 1, las firmas de auditoría deben contar con la información que consideren necesaria respecto a los clientes, con el fin de obtener evidencia frente a la aceptación y/o continuidad del encargo de auditoria. Esta actividad se realiza con el fin de establecer seguridad frente los propietarios de las compañías, al igual permite analizar las condiciones del ente, con el fin de determinar las competencias de los posibles auditores del encargó.

- Realización del Encargo: las actividades de Dirección, supervisión, realización y revisión estarán a cargo del socio, quien asume la responsabilidad global como se mencionó en el literal a, al igual que será el responsable, de que el informe de auditoría sea el adecuado conforme las condiciones del ente auditado. Es indispensable, por lo tanto, dar a conocer a los miembros del equipo temas relacionados con objetivos, naturaleza, posibles riesgos y el detalle de las actividades para la realización del encargo, lo que significa que debe existir una planeación inicial, que deber ser de seguimiento por los auditores, lo que permite la total comunicación fluida entre el equipo auditor y su líder (socio).

- Seguimiento: para la efectividad de la auditoría de calidad, debe establecerse un proceso de seguimiento que proporcione la seguridad razonable de que las políticas y procedimientos. Frente a este aspecto cabe mencionar lo estipulado en al NICC 1 la cual manifiesta que "requiere que la firma de auditoría establezca un sistema de seguimiento diseñado para que proporcionarle una seguridad razonable de que sus políticas $\mathrm{y}$ procedimientos relacionados con el sistema de control de calidad son pertinentes, adecuados y operan eficazmente"

- Documentación: el auditor incluirá en la documentación de Auditoría8 temas relacionados con el cumplimiento de requerimientos éticos y conclusiones frente al cumplimiento de los requerimientos de independencia aplicables al encargo de auditoría. Es 
indispensable entonces que exista una documentación detallada y completa de cada una de las consultas realizadas, recordemos que la documentación es soporte del encargo de auditoría y por lo tanto será la documentación, la prueba de la opinión de los estados financieros auditados.

7. Alcance. trata de las responsabilidades específicas que tiene el auditor en relación con los procedimientos de control de calidad de una auditoría de estados financieros. También trata, cuando proceda, de las responsabilidades del revisor de control de calidad del encargo. Esta NIA debe interpretarse juntamente con los requerimientos de ética aplicables.

\section{Objetivo.}

los objetivos del auditor, cuando va a implementar procedimientos de control de calidad relativos al encargo que le proporcionen una seguridad razonable de que:

- la auditoría cumple las normas profesionales y los requerimientos legales y reglamentarios aplicables.

- el informe emitido por el auditor es adecuado en función de las circunstancias.

\section{Aceptación y continuidad de las relaciones con clientes y de encargos de auditoria}

- El socio del encargo deberá satisfacerse de que se han aplicado los procedimientos adecuados en relación con la aceptación y continuidad de las relaciones con clientes, y de encargos de auditoría, y determinará si las conclusiones alcanzadas al respecto son adecuadas.

- Si el socio del encargo obtiene información que, de haber estado disponible con anterioridad, hubiese sido causa de que la firma de auditoría rehusara el encargo de auditoría, el socio del encargo comunicará dicha información a la firma de auditoría a la mayor brevedad, con el fin de que la firma de auditoría y el socio del encargo puedan adoptar las medidas necesarias.

Asignación de los equipos a los encargos. Se considera miembro del equipo del encargo a una persona especializada en un área específica de contabilidad o auditoría, tanto si ha sido contratada por la firma de auditoría como si trabaja para ella, que aplique procedimientos de auditoría en el encargo. Sin embargo, una persona con dicha especialización no se considera miembro del equipo del encargo si su participación en el encargo se limita a consultas.

Al considerar la competencia y capacidad apropiadas que se espera tenga el equipo del encargo en su conjunto, el socio del encargo puede tener en cuenta en relación con dicho equipo aspectos tales como:

- $\mathrm{Su}$ conocimiento y experiencia práctica en encargos de auditoría de naturaleza y complejidad similares merced a una formación práctica y participación adecuadas.

- $\mathrm{Su}$ conocimiento de las normas profesionales y de los requerimientos legales $\mathrm{y}$ reglamentarios aplicables 
- Su especialización técnica, incluida la relativa a la tecnología de la información relevante y a áreas especializadas de contabilidad o auditoría.

- $\mathrm{Su}$ conocimiento de los sectores relevantes en los que el cliente desarrolla su actividad.

- Su capacidad de aplicar el juicio profesional.

- Su conocimiento de las políticas y procedimientos de control de calidad de la firma de auditoría

\section{APARTE METODOLOGICO.}

El presente esbozo se circunscribió en la revisión documental y teórica, pero, además, en las vivencialidades de los autores, cuyo aporte es definitivo para el constructo. Adicionalmente se desarrolló un focal group con varios expertos contadores, auditores y revisores fiscales quienes aportaron en su punto de vista y enriquecieron la actividad investigativa.

\section{CONCLUSIONES}

$>$ Cuando se va a empezar un trabajo de auditoria, al momento que termine los resultados deben ser confiables $y$ posibilite identificar y valorar los riesgos, el auditor debe tener en cuenta las NIA, dado que, estas normas permiten evidenciar de manera exacta la situación de la empresa y el desempeño contable que ésta posee en el momento de la evaluación, un claro ejemplo es la auditoria de los estados financieros entre muchos otros.

La intención de las NIA de principio y responsabilidades es conseguir seguridad, sobre la correcta preparación de documentos para realizar la auditoria, sin errores materiales, lo cual garantice el menor grado de riesgo posible en el proceso. Esto es posible mediante el estudio previo de la organización y su entorno, así como el funcionamiento, su control y desempeño interno, de esta manera puede determinar los riesgos posibles de incorreciones material o fraude.

Y ahora con la implementación de las NIA dentro de las firmas de auditoría, permite cubrir todos los requerimientos de la normatividad internacional y que esta se convierta en herramienta fundamental para que las firmas brinden un mejor servicio, que mejoren la calidad de su trabajo, el desempeño profesional de sus integrantes, tener una seguridad razonable y buena fe.

La NIA 220 regula la normativa que aplica a todas las organizaciones, independiente a su tamaño, esta norma desglosa la opinión razonable del auditor, frente a las actuaciones profesionales, éticas y regulatorias aplicables a la empresa u organización auditado y emitidas por los entes regulatorios en el país.

Las Normas Internacionales de Auditoría deben ser aplicadas en forma obligatoria, en la auditoría de estados financieros y también, con la adaptación necesaria.

Para finalizar, se establece que se logra un trabajo de calidad, cuando los resultados son satisfactorios, esto quiere decir que cuando el dictamen e útil para la toma de decisiones de los usuarios de la información y el informe es el resultado de un trabajo planeado y 
ejecutado con debido cuidado y cautela, por objetividad e independencia y cumplimiento del código de ética.

\section{BIBLIOGRAFIA}

CAIGG (2015). Diccionario de auditoría interna para el sector público. Recuperado el 6 de enero de 2019, p 5.9, 10,15 de http://www.auditoriainterna degobierno.gob.cl/wpconten t/upLoads/2015/06/DOCU MENTO-TECNICON\%C2\%B0-82DICCIONARIO-AUDINT-PARA-EL-SECTOR PUBLICO.Pdf

Velásquez Luis, (2004) Calidad de la gestión y gestión de la calidad. Recuperado el 15 de enero de 2019, p 1 de https://www.eltiempo.com/ archivo/documento/MAM$\underline{1532168}$

IFAC, Federación Internacional de Contadores (2011). Guía de control de calidad para pequeñas y medianas firmas de auditoría. Recuperado 17 de enero del 2019 p 25 de http://www.imcp.org.mx/I MG/pdf/GUIA_DE_CONT ROL_DE_CALIDAD_PAR A_PEQUENAS_Y_MEDI ANAS_FIRMAS_DE_AU DITORIA_3a_edicion.pdf

Escobar Naira, (2015) Enfoque de control de calidad de la auditoria de estados financieros en las pymes en
Colombia. Recuperado el 29 de enero del 2019, P 5. De https://repository.unimilitar. edu.co/bitstream/handle/106 54/7527/ESCOBARSANC HEZNAIRAALEXANDRA 2015.pdf;jsessionid=7EE8A FC706C0C45A9BE54E0D DDCE9F20?sequence $=1$.pd $\mathrm{f}$

Urbano Cesar, (2011) Certificación de control de calidad para las firmas auditoras peruanas. Recuperado el 20 de diciembre 2019, p 3 http://revistasinvestigacion. unmsm.edu.pe/index.php/qu ipu/article/view/3721/2988. Pdf

Pizarro Elizabeth, Pozo Sergio, Díaz Adis (2017) El control de calidad en empresas auditoras pequeñas $y$ medianas en Manabí, Ecuador. Recuperado 13 de enero del 2019 de http://scielo.sld.cu/scielo.ph p? script $=$ sci_arttext\&pid $=S$ 207360612017000200023.art

Corredor Nelson, Piragauta Carlos (2015) Practicas de control de calidad que aplican las firmas de auditoría respecto a la independencia de los auditores frente a sus auditados. Recuperado el 21de diciembre del 2018, p 9 de https://expeditiorepositorio. utadeo.edu.co/bitstream/han dle/20.500.12010/2491/Pr\% C3\%A1cticas\%20de\%20co 
ntrol $\% 20$ de $\% 20$ calidad $\% 20$ que $\% 20$ aplican $\% 201$ as $\% 20 \mathrm{f}$ irmas $\% 20$ de $\% 20$ auditor\%C $3 \% \mathrm{ADa} \% 20$ respecto\%20a $\% 201 a \% 20$ independencia\% 20d.pdf?sequence $=1 \&$ isAll owed=y.Pdf

Estrada Jorge (2015), Sistemas de gestión de la calidad: una herramienta imprescindible en la auditoría financiera. Recuperado el 17 de enero 2019 p 8 de http://ojs.tdea.edu.co/index. php/encontexto/article/view /297.Pdf

García Dora, Montaño Carlos, Ruiz Juan (2016) La auditoría y su control de calidad: una mirada desde las normas de aseguramiento de la información, en Colombia. Recuperado el 19 de enero del 2019 p 1 de http://revistas.ugca.edu.co/i ndex.php/contexto/article/vi ew/652/1016.

Borràs Frederic, (1914) El reto de mejorar la calidad de la auditoría, en Barcelona. Recuperado 28 de enero del 2019 p 15,16 de https://raed.academy/wpcontent/uploads/2017/02/El -reto-de-mejorar-la-calidadde-laauditor\%C3\%ADa.pdf.

IFAC. (2009). Código de Ética para los profesionales de la contabilidad (Tercera ed.). (Auditores, Trad.) España. Recuperado el 12 de 01 de 2019, https://www.ifac.org/system /files/publications/files/codi go-de-etica-paraprofesionales-de-lacontabilidad.pdf

Ibarra \& Asociados, a. c. (s.f.). Auditool. Recuperado el 2 de octubre de 2015, de Red Global de conocimientos de Auditoria y Control Interno: 24

http://www.auditool.org/blo g/auditoria-externa/1094nias-normasinternacionalesde-auditoria-yaseguramiento

Castro, L. (2015). Normas Internacionales de Auditoria (NIAS) Metodología y análisis. Ensayo para optar el título de contador, 1-27.

Bogotá, Colombia: Universidad MIitar Nueva Granada. Recuperado el 12 de 01 de 2019, de https://repository.unimilitar. edu.co/bitstream/handle/106 54/6842/ensayo.pdf?sequen $\underline{\mathrm{ce}}=1$

Mantilla, S. (2002). Código de Ética de La IFAC. ( traducido al español- departamento de ciencias contables- unive rsidad Javeriana, https://www.gestiopolis.co $\mathrm{m} /$ codigo-de-etica-de-laifac/

IFAC, Federación Internacional de Contadores (2010). Glosario. En F. I.

http://www.ifac.org/system/files/pu blications/files/ES_A0_IPS ASB Handbook 2011 Vol 
ume I\%20(ALL)\%20-

\%20Final 1.pdf

Escobar N. Enfoque de control de calidad de la Auditoria de estados financieros en las Pymes de Colombia. Especialización en revisoría fiscal

https://repository.unimilitar. edu.co/bitstream/handle/106 54/7527/ESCOBARSANC HEZNAIRAALEXANDRA 2015.pdf;jsessionid $=48 \mathrm{C} 89$ 45D9B639679AF5C0E0D

AD5C920E?sequence $=1$

Ruiz Y. Las Normas Internacionales de Auditoría, herramienta fundamental en las firmas de auditoría.

https://repository.unimilitar.edu.co/ bitstream/handle/10654/152 60/RuizDiazYohannaAndre a2016.pdf.pdf? sequence $=1$ \&isAllowed $=\mathrm{y}$

Instituto Mexicano de Contadores Públicos (2010), control de calidad para una auditoria de estados financieros.

http://imcp.org.mx/wpcontent/uploads/2013/01/NI A 220.pdf

Control De Calidad De La Auditoría De Estados Financieros NiaEs 220(2013, p 12)http://www.icac.meh.es/ NIAS/NIA\%20220\%20p\% 20def.pdf 